\title{
EL SUBSISTEMA DE FORMACIÓN CONTABLE EN COLOMBIA Y MÉXICO*
}

\author{
NOEMÍ VÁSQUEZ QUEVEDO** \& RUTH ALEJANDRA PATIÑO JACINTO*** \\ INSTITUTO TECNOLÓGICO Y DE ESTUDIOS SUPERIORES DE MONTERREY (MÉXICO) \\ UNIVERSIDAD SANTO TOMÁS (COLOMBIA)
}

Recibido/ Received/ Recebido: 04/02/2014 - Aceptado/ Accepted / Aprovado: 12/08/2014

\begin{abstract}
Resumen
Este artículo de investigación muestra las características del subsistema de formación contable (Jarne, 1997) en Colombia y México, cuyo proceso de convergencia internacional inició en los años 2009 y 2005, respectivamente. A través de una revisión documental y bibliográfica, se señalan lineamientos de formación contable que establecen organismos nacionales de cada país e internacionales como la UNCTAD y el IFAC, describiendo la situación actual de los países con respecto a los requerimientos de educación, desarrollo de contadores profesionales y de educación profesional continua. El estudio muestra como principales diferencias entre los dos países, la convergencia internacional en temas de formación contable y la certificación de profesionales; y como coincidencia, la necesidad de incluir mayor investigación en los planes de estudio.
\end{abstract}

Palabras clave: Formación contable, Sistemas contables, Convergencia internacional.

\section{THE SUBSYSTEM OF ACCOUNTANT TRAINING ON COLOMBIA AND MEXICO}

\begin{abstract}
This article shows characteristics of research of the subsystem accounting training (Jarne, 1997) in Colombia and Mexico, which process of international convergence began in the year 2009 and 2005, correspondingly. Through a documental revision and bibliographical, it's been pointed out guidelines of accounting training that are establish in national organisms of each country and international like UNCTAD and the IFAC, describing the current situation of the countries with respect to the requirements of education, development of accounting professionals and the continuing professional education. The study shows how the principle differences between the two countries, the international convergence in themes that show accounting training and the certification of professionals; and as a coincidence, the necessity of including mayor research in the plans of study.

Keywords: Accountant formation, Accountant system, International convergence.
\end{abstract}

" Artículo de investigación derivado del proyecto "Comparación de los subsistemas contables de Colombia y México", realizado en el año 2013, cofinanciado por la Universidad Santo Tomás y el Instituto Tecnológico y de Estudios Superiores de Monterrey - ITESM.

* Profesora investigadora de tiempo completo en el ITESM, Campus Ciudad de México. Contadora Pública, Instituto Tecnológico y de Estudios Superiores de Monterrey - ITESM, Campus Monterrey. Maestra en finanzas y Doctora en Administración, Instituto Tecnológico y de Estudios Superiores de Monterrey - ITESM, Campus Ciudad de México. Correo electrónico: nvasquez@itesm.mx. Tel: (52) 5554832257. Dirección postal: Calle del Puente 222, Colonia Ejidos de Huipulco, Tlalpan. C.P. 14380, México D. F.

*** Coordinadora del Centro de Investigaciones de Contaduría Pública (CICOP), Universidad Santo Tomás. Directora del Observatorio Colombiano de Educación Contable (OCEC). Contadora Pública, Universidad Nacional de Colombia. Magíster en Ciencias Económicas, Universidad Santo Tomás. Líder del grupo de investigación "Contaduría: información, control e impacto social". Correo electrónico: alejandrapatino@usantotomas.edu.co. Tel. (57) (1) 5878797 Ext. 1403. Dirección postal: Cra 9 \# 51-11, Bogotá. 


\title{
O SUBSISTEMA DE FORMAÇÃO CONTÁBIL NA COLÔMBIA E NO MÉXICO
}

\begin{abstract}
Resumo
Este artigo de pesquisa mostra as características do subsistema contábil de formação (Jarne, 1997) na Colômbia e no México, cujo processo de convergência internacional iniciou nos anos 2009 e 2005, respectivamente. Através de uma revisão documental e bibliográfica, assinalam-se alinhamentos de formação contábil que estabelecem organismos nacionais de cada país e internacionais como a UNCTAD e o IFAC, descrevendo a situação atual dos países com respeito aos requerimentos de educação, desenvolvimento de contadores profissionais e de educação profissional contínua. O estudo mostra como principais diferenças entre os dois países, a convergência internacional em temas de formação contábil e a certificação de profissionais; e como coincidência, a necessidade de incluir maior pesquisa nos planos de estudo.
\end{abstract}

Palavras chave: Formação contábil, Sistemas contábeis, Convergência internacional.

Vásquez, N. \& Patiño, R. (2015). El subsistema de formación contable en Colombia y México. En: Revista de la Facultad de Ciencias Económicas de la Universidad Militar Nueva Granada. rev.fac. cienc.econ, XXIII (1).

JEL: M41, M48.

\section{Introducción}

La última década del siglo XX se caracterizó por una fuerte competencia a nivel mundial enfocada principalmente en la reducción de costos innecesarios, la orientación al cliente y la implantación de sistemas para medir e incrementar la generación de valor de las organizaciones. En el inicio de la segunda década del siglo XXI, términos como globalización, competencia y sistemas de información, forman parte de la cotidianidad de las empresas.

Dentro de las principales influencias que ha generado la globalización, se encuentran el incremento de la responsabilidad e involucramiento de los empleados, el surgimiento de organizaciones de servicios, el incremento de la velocidad de los cambios, el incremento de la innovación y principalmente, el incremento de la competencia global (Ramírez, 2013).

Como consecuencia de este último, la apertura a los mercados globalizados se ha hecho inminente, así como la necesidad de generar información financiera que sea confiable para inversionistas y otros involucrados en decisiones económicas.
Con la finalidad de generar confianza en los reportes financieros, se ha establecido como un mecanismo prominentemente importante, el regulatorio. De ahí surge la necesidad de establecer las normas internacionales de contabilidad, y posteriormente las Normas Internacionales de Información Financiera (IFRS, por sus siglas en inglés), aunque esta situación ha sido cuestionada.

Este entorno normativo exige mayor capacitación del recurso humano y por ello este trabajo tiene como objetivo describir los pronunciamientos que en materia de formación contable y en el ejercicio de la profesión, señalan los principales organismos internacionales relacionados con el tema; así como describir la situación en la que se encuentra la educación contable en Colombia y México.

Joshi, Takara, Gremone \& González (2012) señalan que la implementación de las IFRS, usadas por más de 120 países alrededor del mundo, ha generado retos de distinta índole, tales como de técnica contable, sistema tributario, procesos de negocio, sistemas de información y recursos humanos. Vásquez (2010) analiza el proceso de adopción de IFRS en México 
y encuentra que como resultado de encuestas aplicadas por firmas internacionales de auditoría en el 2009 y 2010, los tres principales retos identificados en la transición a IFRS fueron: i) la falta de guías técnico-contables; ii) la falta de personal calificado; $y$ iii) el hecho de que el tema todavía no era de prioridad para la alta dirección. De hecho, la falta de personal calificado fue el segundo reto más importante identificado en 2009 y el tercero en 2010.

Vásquez (2010) también identifica las áreas más afectadas en cuanto al grado de dificultad que experimentaron en el proceso de adopción y señala que hay un incremento importante de ejecutivos que perciben al área de capacitación y recursos humanos como la más afectada, colocándola en segundo lugar por debajo de la contabilidad técnica, en la cual se tiene la percepción de mayor afectación.

Por otra parte, Chiquiar (2010) señala como principales retos de la adopción de IFRS en Latinoamérica, el desconocimiento de la normatividad internacional, la resistencia al cambio, la reciente obligatoriedad, los costos de implementación y la falta de atención en la educación superior.

Si bien el uso de estándares internacionales es ineludible, también lo es la capacitación del recurso humano en el conocimiento, implantación y evaluación de dichas normas. En el ámbito internacional la preocupación en este sentido se ha manifestado y se están haciendo estudios y propuestas para atender la necesidad de desarrollo de capital humano. El Grupo Intergubernamental de Trabajo de Expertos en Normas Internacionales de Contabilidad y Presentación de Informes (ISAR, por sus siglas en inglés), perteneciente a la Conferencia de las Naciones Unidas sobre Comercio y Desarrollo (UNCTAD, por sus siglas en inglés) y el Consejo de Normas Internacionales de Formación en Contaduría (IAESB, por sus siglas en inglés), organización independiente dentro del seno de la Federación Internacional de Contabilidad (IFAC, por sus siglas en inglés); se han ocupado de evaluar las tendencias y los retos que están presentándose en los distintos países en cuanto al desarrollo de recursos humanos en el ámbito contable.

1 Fuente: Patiño \& Vásquez (2013).
Como resultado de su labor en este sentido, la UNCTAD ha emitido una propuesta de Modelo de Currículo de Contabilidad con el propósito de describir a la comunidad internacional, las áreas técnicas que un individuo debe conocer para ser considerado un profesional contable. Por su parte, el ISAR ha elaborado un instrumento de desarrollo contable (IDC) que promueva la presentación de información empresarial de alta calidad. El IDC consta de cuatro pilares: legal y reglamentario, institucional, de capacidad humana y de proceso de fomento de la capacidad (UNCTAD, 2013).

Por su parte, el IAESB en su labor de brindar estándares de formación, ha emitido ocho Estándares Internacionales de Formación Contable (IES, por sus siglas en inglés) que abordan los principios de aprendizaje y desarrollo para los contadores profesionales. Tales normas están conformadas por aquéllas que indican los requerimientos de ingreso a la educación profesional en contaduría y las que señalan los requerimientos relativos al ingreso a la profesión.

Ante el entorno global de estándares contables y normas de educación internacionales, en este artículo el enfoque se centra en revisar la situación actual en materia de capacidad humana en Colombia y México, así como en identificar los retos principales a los que se enfrentan en el corto y mediano plazo de acuerdo con las necesidades del mercado, pero también de conformidad con los lineamientos de organismos nacionales e internacionales.

Lo anterior desde la perspectiva propuesta por Jarne (1997) quien conceptualiza a los sistemas contables y los clasifica en los subsistemas que se muestran en la Tabla 1.

Tabla 1. Subsistemas propuestos por Jarne $(1997)^{1}$

\begin{tabular}{|c|l|}
\hline Subsistema & \multicolumn{1}{|c|}{ Concepto } \\
\hline Regulador & $\begin{array}{l}\text { Los aspectos relacionados con la gestación, elabora- } \\
\text { ción, perfeccionamiento e implantación de la normativa } \\
\text { contable que, conjugando en el proceso de genesis de } \\
\text { la norma las inquietudes y necesidades de los diferentes } \\
\text { agentes afectados por la misma, va a regir la generación } \\
\text { y publicación de la información contable empresarial. }\end{array}$ \\
\hline
\end{tabular}




\begin{tabular}{|c|c|}
\hline $\begin{array}{l}\text { Principios } \\
\text { Contables }\end{array}$ & $\begin{array}{l}\text { La parte del sistema contable que, a través de la de- } \\
\text { finición de unos conceptos contables fundamentales } \\
\text { (PCGA), traza la línea a seguir para la obtención de } \\
\text { unos estados financieros que reflejen la imagen fiel } \\
\text { del patrimonio, situación financiera y resultados de la } \\
\text { empresa. }\end{array}$ \\
\hline Profesional & $\begin{array}{l}\text { La parte del sistema contable que comprende la acti- } \\
\text { vidad realizada por los profesionales de la contabilidad } \\
\text { y la auditoría, compaginando la protección del interés } \\
\text { público y la satisfacción de las necesidades de sus } \\
\text { clientes, así como la actuación de las organizaciones } \\
\text { de profesionales de la contabilidad y de las multinacio- } \\
\text { nales de auditoria. }\end{array}$ \\
\hline $\begin{array}{l}\text { Formación } \\
\text { Contables }\end{array}$ & $\begin{array}{l}\text { La parte del sistema contable encargado del desarrollo } \\
\text { de los conocimientos y técnicas básicas para aplicar } \\
\text { los principios y normas contables, y transmitirlas a un } \\
\text { conjunto de agentes que sean capaces de aplicarlas } \\
\text { con posterioridad (profesionales), así como, de la es- } \\
\text { tructuración del recorrido lógico para la obtención, por } \\
\text { parte de los profesionales, de la cualifiación profesional } \\
\text { (expertos contables). }\end{array}$ \\
\hline $\begin{array}{l}\text { Prácticas } \\
\text { Contables }\end{array}$ & $\begin{array}{l}\text { Prácticas de valoración: la parte del sistema contable } \\
\text { que refleja los criterios objetivos seguidos en el proce- } \\
\text { so de reconocimiento, medición y valoración contables, } \\
\text { poniendo en condiciones de ser incluidos en los es- } \\
\text { tados e informes financieros, en términos monetarios, } \\
\text { a los flujos económicos, derechos, obligaciones y de- } \\
\text { más modificaciones en la estructura patrimonial de la } \\
\text { empresa surgidos de las transacciones realizadas por } \\
\text { la misma. } \\
\text { Prácticas de información: La parte del sistema conta- } \\
\text { ble encargado de determinar la cantidad y formato de } \\
\text { la información contable, previamente procesada, que se } \\
\text { va a suministrar al exterior, así como la periodicidad y } \\
\text { mecanismos de dicha comunicación. }\end{array}$ \\
\hline
\end{tabular}

De tales subsistemas, en este trabajo se estudiará el correspondiente al de formación, dando continuidad al trabajo realizado por Patiño \& Vásquez (2013) en el que identifican las características que Colombia y México presentan de cada uno de estos subsistemas.

La selección de estos dos países obedece a que se trata de países latinoamericanos que han decidido adoptar las IFRS, con un referente histórico en materia contable caracterizado por una formación integral, interdisciplinaria, preocupada por profundizar para especializar, por acreditar instituciones educativas y contar con gremios profesionales involucrados en el proceso formativo. Adicionalmente, existe el interés de profundizar en el subsistema relativo a educación que se inició en la investigación antes mencionada. Por otra parte, se identificaron semejanzas y diferencias entre ambos países que resultan interesantes de relacionar con su avance en capacitación del desarrollo humano. Por un lado, en ambos países la decisión de adoptar las IFRS fue por iniciativa del gobierno; sin embargo, en Colombia, las normas contables locales han sido elaboradas por un organismo gubernamental (CTCP), mientras que en México las ha realizado un organismo privado (CINIF). En cuanto a las empresas a las que se ha convocado para implementar IFRS, en el caso de Colombia han sido más incluyentes ya que todas las empresas, incluyendo las PYMES, están obligadas a adoptar IFRS de manera escalonada; mientras que en México sólo han sido requeridas las empresas que cotizan en la Bolsa Mexicana de Valores.

En cuanto a revisiones previas sobre el tema referente (sistemas contables), se encuentran diversos autores, Doupnik \& Salter (1993) identifica como los principales autores a Mueller (1967; 1968), Seidler (1967), AAA (1977), Waterhouse International (1973; 1975; 1979), Da Costa et al. (1978), Frank (1979), Nair \& Frank (1980), Nobes (1981), Stewart (1981), Nair (1982), Nobes (1987), y en contextos latinoamericanos aparecen: Jarne (1997); Callao \& Jarne (1995); Laínez \& Jarne (1999); Cañibano \& Mora (1999); Rodríguez (s.f.); García \& Garrido (1995); García \& Gandía (1998); García, Laínez \& Monterrey (1995); Laínez, et al. (2001); Zorio, García \& Pucheta (2004); donde el más cercano al contexto latinoamericano es el realizado por Morales \& Jarne (2006), el cual se realizó en Argentina, Brasil y Chile. De igual forma han indagado sobre el tema de educación contable en cada uno de los países, sin embargo en el caso específico de revisión a partir del concepto de subsistema de educación en comparativo no se encontró revisiones previas.

Así pues, resulta interesante indagar y sugerir si las similitudes y diferencias entre ambos países, guardan alguna relación con las decisiones que éstos han tenido en materia de formación contable y desarrollo profesional continuo. 
El artículo está organizado de la siguiente manera: la sección 2 presenta la metodología y fuentes de información, la sección 3 conceptualiza el subsistema contable de formación de acuerdo con Jarne (1997), la sección 4 muestra un análisis comparativo del Subsistema Contable de Formación con las propuestas de organismos internacionales, y finalmente la sección 5 presenta las conclusiones del estudio.

\section{Metodología}

El trabajo se realiza a partir de una revisión documental y bibliográfica del tema en cuestión nombrado por distintos autores y fuentes de referencia, como formación o educación contable, desarrollo de habilidades profesionales o de capacidad humana, todos éstos relacionados con el ámbito contable (Martín, 2000; Martínez, 2007; Montoya \& Farías, 2011; UNCTAD, 2013).

Dado que las fuentes de información primordialmente son documentales (primarias), consideramos importante presentar su clasificación y describir brevemente cada una de ellas (Tabla 2 ).

Tabla 2. Fuentes de información ${ }^{2}$

\begin{tabular}{|l|l|}
\hline \multicolumn{1}{|c|}{ Tipo } & \multicolumn{1}{|c|}{ Descripción } \\
\hline Bibliográficas & $\begin{array}{l}\text { Libros, principalmente de contabilidad. } \\
\text { Revistas científicas: Journals contables y de } \\
\text { educación. } \\
\text { Revistas de divulgación contable. }\end{array}$ \\
\hline Organismos Profesionales & $\begin{array}{l}\text { Colombia: } \\
\text { Consejo Técnico de la Contaduría Pública. } \\
\text { México: } \\
\text { Instituto Mexicano de Contadores Públicos } \\
\text { (IMCP): Organismo nacional que agremia a } \\
\text { la profesión contable. }\end{array}$ \\
\hline Organismos de Educación & $\begin{array}{l}\text { Colombia: } \\
\text { Ministerio de Educación Nacional. } \\
\text { México: } \\
\text { Asociación Nacional de Facultades y Es- } \\
\text { cuelas de Contaduría y Administración (AN- } \\
\text { FECA): Institución de orientación y servicio } \\
\text { académico en las carreras de negocios. } \\
\text { Secretaría de Educación Pública (SEP): } \\
\text { Organismo gubernamental encargado del } \\
\text { sistema educativo nacional. }\end{array}$ \\
\hline
\end{tabular}

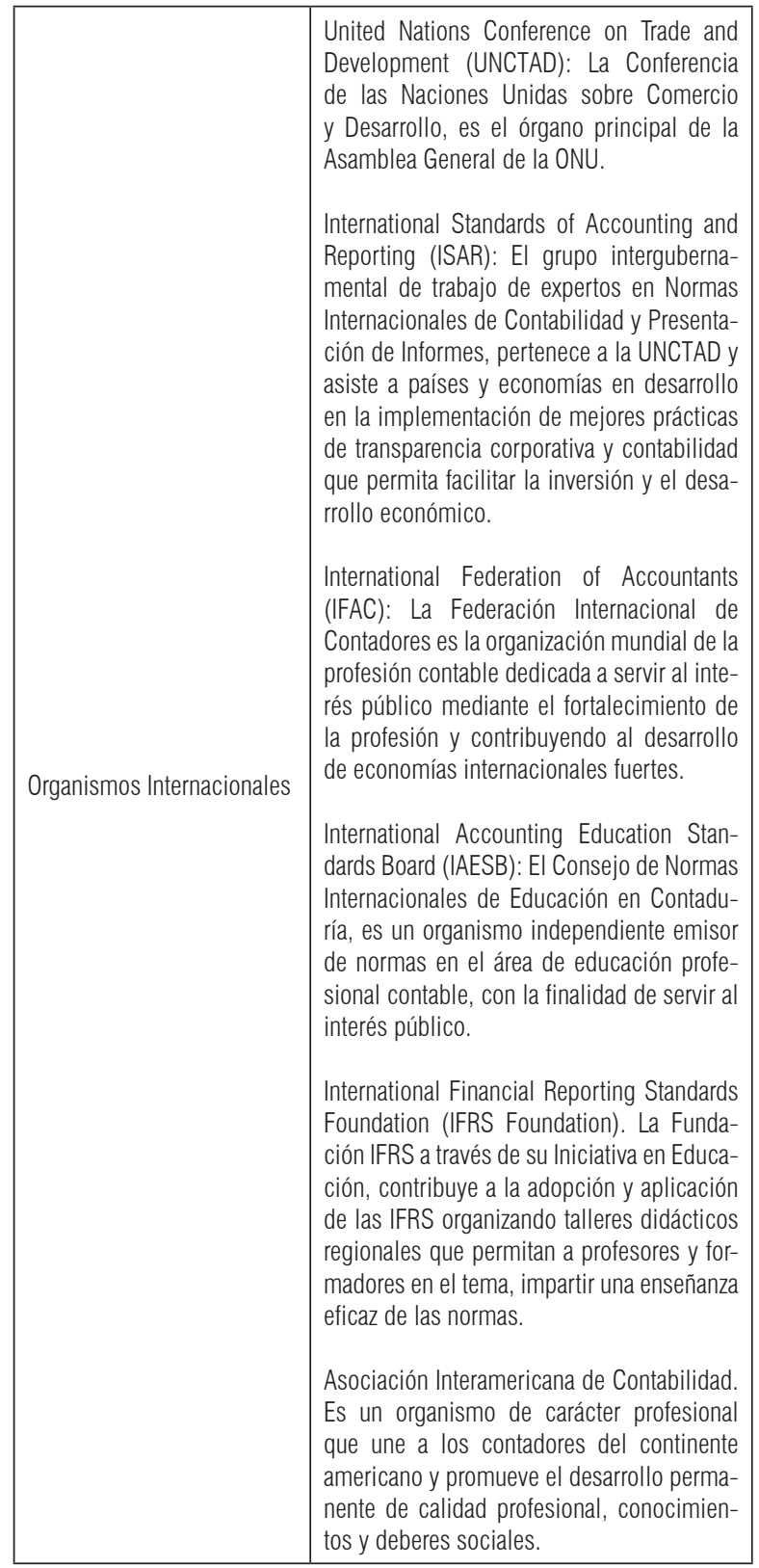

\section{Conceptualización del Subsistema Contable de Formación}

La definición que presenta Jarne (1997) del subsistema contable de formación, es la de ser "el encargado de dotar del capital humano necesario al resto

\footnotetext{
2 Fuente: Elaboración propia.
} 
del sistema contable, posibilitando su desarrollo" y extiende el concepto de la siguiente manera: "la parte del sistema contable encargado del desarrollo de los conocimientos y técnicas básicas para aplicar los principios y normas contables, y transmitirlas a un conjunto de agentes que sean capaces de aplicarlas con posterioridad (profesionales), así como, de la estructuración del recorrido lógico para la obtención, por parte de los profesionales, de la cualificación profesional (expertos contables)".

En general tiene gran importancia, ya que da soporte para que los demás subsistemas funcionen, se crea capital humano que conozca las características del entorno y sea propositivo ante las situaciones problemáticas que se dan en la actualidad.

Es así como, de acuerdo con Jarne (1997) se incluyen funciones del subsistema contable como: ser la base para elaborar normativa contable, suministrar la base conceptual a los encargados de decidir en materia de principios contables y aportar profesionales que evolucionen junto con las necesidades del entorno.

De tal forma que se puede evidenciar, de acuerdo con Patiño Jacinto \& Vásquez Quevedo (2013), que:

"El subsistema de formación es uno de los más relevantes en contabilidad, ya que contempla cómo la educación profesional responde a las necesidades del entorno, no sólo a las correspondientes a las empresas, sino a las necesidades sociales en general. Sin embargo, se investiga separado de contextos como el profesional o el regulatorio, dado lo anterior existe la necesidad urgente de vincular el estudio de la educación a las situaciones del ámbito contable".

En general, como lo menciona Jarne (1997), la formación se encarga de que los profesionales cumplan con los requisitos del entorno, lo cuales van a depender de los objetivos, dichos objetivos son referentes al modelo educativo de cada país, allí se encuentran algunas tendencias, un modelo aplicado en la actualidad es el de competencias, impulsado por instituciones internacionales como "la Comisión Económica para América
Latina [CEPAL], la Organización para la Cooperación y el Desarrollo Económico [OECD], el Proyecto Tuning, International Federation of Accountants [IFAC]" (Valero, Patiño \& Duque, 2013), en general las competencias buscan que los egresados se desempeñen de forma eficiente en el entorno globalizado, lo cual ha sido ampliamente cuestionado ya que desde otras miradas esto hace que se pierda el carácter crítico y diverso de la educación.

El subsistema contable incluye diversos aspectos, dentro de los que evalúa Jarne (1997) aparecen:

- La relación entre universidad y empresa en términos de trabajos conjuntos.

- El reconocimiento de un organismo oficial para la certificación profesional.

- La existencia de formación continua por parte de las firmas de auditoría.

- La participación de la educación contable por parte de universidades públicas y privadas.

- La valoración de docencia frente a investigación.

- Realización de trabajos en la universidad dirigidos al ámbito empresarial.

- Formación contable en las empresas.

- Rotación de los profesores por diversas universidades.

- Años de exigencia para desempeñarse en auditoria.

- Financiación privada en la labor investigadora.

- Exigencia de edad mínima para acceder a la obtención de cualificación profesional.

En general estos aspectos evalúan cómo todos los aspectos relacionados con la docencia impactan en la profesión contable y en todo el sistema contable. El desarrollo de la contabilidad se da por medio de la investigación y de procesos de reproducción del conocimiento, lo cual impacta la regulación, los procesos de valoración, entre otros.

\section{Análisis comparativo del Subsistema Contable de Formación con las propuestas de organismos internacionales}

Uno de los principales organismos que reglamenta la educación contable internacionalmente es la $\mathrm{Fe}$ - 
deración Internacional de Contadores (IFAC por sus siglas en inglés), la cual además de generar estándares en contabilidad gubernamental, en auditoría y ética, aporta aspectos trascendentales en formación por competencias, que dependiendo del enfoque educativo, han sido objetos de críticas o han sido adoptadas en los modelos de los países.

La IFAC emite los Estándares Internacionales de Educación Contable, los cuales de acuerdo con (Patiño \& Santos, 2009) incluyen: requisitos de admisión, educación general, educación profesional, evaluación y requisitos de experiencia. Todo ello a partir de una visión del profesional contable bajo la incidencia de los estándares internacionales de contabilidad y de información financiera IAS/IFRS, aunque incluye aspectos como el trabajo en grupo y las habilidades comunicativas, además de conocimientos en finanzas, administración, entre otros.

De acuerdo con Patiño \& Valero (2013), los referentes más recientes de esta institución son: el manual de pronunciamientos internacionales en formación (año 2010) y mejora de la educación contable profesional (año 2012), además se menciona que en los mismos aparecen diversos conceptos (Tabla 3).

Tabla 3. Conceptos incluidos en los Estándares Internacionales de Formación Contable ${ }^{3}$

\begin{tabular}{|l|l|}
\hline \multicolumn{1}{|c|}{ Palabra } & Concepto dado por IFAC \\
\hline Competencia & $\begin{array}{l}\text { La capacidad de realizar una función de trabajo definido } \\
\text { conforme a un estándar con referencia a los ambientes } \\
\text { de trabajo. Para demostrar competencia en un rol es- } \\
\text { pecífico, un contador profesional debe poseer: (a) los } \\
\text { conocimientos profesionales, (b) las cualificaciones } \\
\text { profesionales, y (c) los valores, la ética y actitudes pro- } \\
\text { fesionales. }\end{array}$ \\
\hline Educación & $\begin{array}{l}\text { Un proceso sistemático destinado a la adquisición y } \\
\text { desarrollo de conocimientos, habilidades y otras ca- } \\
\text { pacidades en los individuos, un proceso que es nor- } \\
\text { malmente (no exclusivamente) realizado en entornos } \\
\text { académicos. }\end{array}$ \\
\hline $\begin{array}{l}\text { Experiencia } \\
\text { Práctica }\end{array}$ & $\begin{array}{l}\text { Se refiere a las actividades de trabajo que son relevantes } \\
\text { para desarrollo de la competencia. }\end{array}$ \\
\hline
\end{tabular}

3 Fuente: Patiño \& Valero (2013) a partir de IFAC (2010).

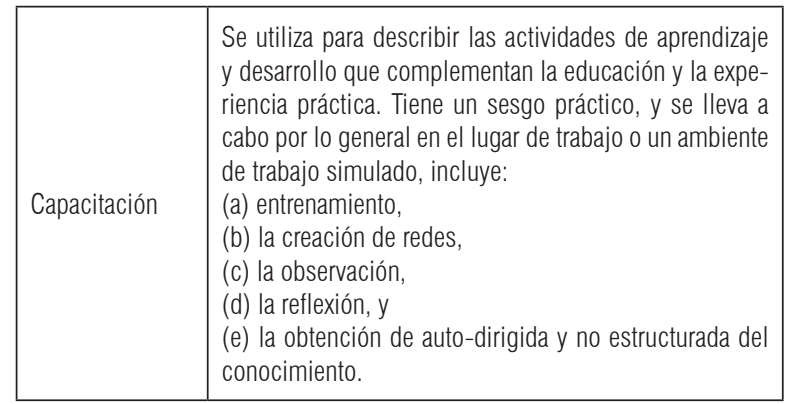

La UNCTAD (Conferencia de las Naciones Unidas para el Comercio y el Desarrollo), es otro organismo que ha promovido a la comunidad internacional, los puntos de referencia de características de un contador profesional que le permitan ofrecer un mejor servicio ante una economía globalizada. Dicho organismo presentó una propuesta de plan de estudios (Model Accounting Curriculum, MC) para un contador profesional en 1999 (Patiño \& Santos, 2009) y una nueva versión en el 2011 (UNCTAD, 2011). Este modelo presenta un plan de estudios detallado que describe los temas técnicos que un individuo debe dominar para convertirse en un profesional de la contabilidad.

El Consejo de Normas Internacionales de Formación en Contaduría (IAESB), creado en 2005 en sustitución de la Comisión de Educación de la Federación Internacional de Contadores (IFAC), ha emitido ocho normas internacionales de formación en contaduría (IES, por sus siglas en inglés). Los temas de estas normas consisten en: requisitos de ingreso; contenido de los programas profesionales de formación en contaduría; habilidades profesionales y formación general; valores ética y actitud profesionales; requisitos de experiencia práctica; evaluación de las capacidades y competencia profesional; desarrollo profesional continuo; y requisitos de competencia que deben reunir los auditores profesionales (UNCTAD, 2013).

Otros organismos que fomentan la capacitación en contabilidad, son la Fundación IFRS y la Asociación 
Interamericana de Contabilidad. La fundación IFRS a través de la iniciativa en materia de educación, ofrece capacitación que permita adoptar y aplicar de manera coherente las IFRS. Así pues, ofrece talleres didácticos en diferentes regiones del mundo que preparen a formadores de IFRS de forma tal que sean capaces de otorgar una enseñanza eficaz de la normatividad internacional (UNCTAD, 2013). Por su parte, la Asociación Interamericana de Contabilidad promueve también la educación en el área contable a través de su comisión técnica de educación integrada por más de 12 países latinoamericanos. Su más reciente contribución ha sido la Certificación Interamericana en las NIIF para las Pymes (AIC, 2013).

El aporte de esta sección es presentar un análisis comparativo de las características del subsistema de formación contable propuesto por Jarne (1997) con respecto a los señalamientos que ha emitido la UNCTAD y el IAESB, así como describir la situación relativa en la que se encuentra la educación contable en Colombia y México.

Como se puede apreciar en la Tabla 4, las tres fuentes de referencia hacen una distinción del desarrollo de un contador, entre la formación durante y posterior a los estudios universitarios. Por otra parte, identificamos trabajo en conjunto entre el IFAC y la UNCTAD desde hace varios años y son referencias mutuas en diferentes pronunciamientos y documentos emitidos (UNCTAD-ISAR, 1999). A continuación se describen las características y lineamientos que señalan las referencias del estudio.

\subsection{Formación académica hasta la obtención de la graduación universitaria}

Jarne (1997) define las características posibles de la formación académica universitaria, pudiendo ser entre otras, si la formación es ofrecida por instituciones privadas o públicas, el nivel de demanda de estudios universitarios, las características del profesorado, la inclusión de contabilidad internacional en los planes de estudios y el nivel de colaboración entre la universidad y la empresa.

La descripción de tales características son presentadas por Patiño \& Vásquez (2013) para el caso de
Colombia y México. Cabe señalar que Jarne (1997) no sugiere el contenido técnico contable ni de habilidades que debe ser incluido en un plan de estudios. De las características mencionadas, las dos últimas (1.1.5 y 1.1.6) son referenciadas también por la UNCTAD y el IAESB.

Por su parte, los planes de estudio de los organismos internacionales incluyen como elemento importante en la formación técnica contable, el conocimiento del marco conceptual de las normas internacionales y la preparación de los estados financieros utilizando las IFRS (UNCTAD, 2011; IAESB, 2013).

Por otro lado, el modelo de plan de estudios en contaduría (MC) sugerido por la UNCTAD, 2011, alienta la inclusión del aprendizaje basado en la experiencia a través de un internado (internship) en una firma de contadores públicos, empresa o alguna otra organización independiente a la universidad.

En el caso de México, existen Instituciones de Educación Superior (IES) que incluyen este tipo de ejecución práctica en sus planes de estudios, pero no es un requisito obligatorio ni para las IES ni para los estudiantes (ANFECA, 2011).

\subsection{Plan de estudios de contabilidad global y otros requisitos de calificación, IDC y requisitos para obtener la calificación de contador profesional}

Estos lineamientos dictados por la UNCTAD y el IAESB, incluyen los contenidos técnicos y desarrollo de habilidades que se sugieren para un programa en contaduría.

Por su parte, el Grupo Intergubernamental de Trabajo de Expertos en Normas Internacionales de Contabilidad y Presentación de Informes (ISAR, por sus siglas en inglés) ha elaborado un Instrumento de Desarrollo Contable (IDC, inciso 2.2 en Tabla 4) que incluye una prueba piloto, también conocido como cuestionario, en donde a través de cuatro pilares, se evalúan indicadores que midan la capacidad de los países, de presentar información empresarial de alta calidad (UNCTAD-ISAR, 2010). 
Tabla 4. Análisis comparativo de las características del subsistema de formación contable con los lineamientos internacionales ${ }^{4}$

\begin{tabular}{|c|c|c|}
\hline Jarne (1997) & UNCTAD & IAESB \\
\hline $\begin{array}{l}\text { 1.1 Formación académica hasta la ob- } \\
\text { tención de la graduación universitaria: } \\
\text { 1.1.1 Formación universitaria: sector públi- } \\
\text { co o privado. } \\
\text { 1.1.2 Demanda de estudios universitarios. } \\
\text { 1.1.3 Educación contable con respecto a } \\
\text { demandas sociales. } \\
\text { 1.1.4 Características del profesorado. } \\
\text { 1.1.5 Contabilidad internacional en planes } \\
\text { de estudios. } \\
\text { 1.1.6 Colaboración universidad-empresa. }\end{array}$ & $\begin{array}{l}\text { 2.1 Plan de estudios de contabilidad global y otros } \\
\text { requisitos de calificación: } \\
\text { 2.1.1 Conocimientos y habilidades generales. } \\
\text { 2.1.2 Educación técnica profesional: Modelo de plan de } \\
\text { estudios de contabilidad (MC, por sus siglas en inglés). } \\
\text { 2.2 Instrumento de Desarrollo Contable (Pilar C } \\
\text { referido a capacidad humana). } \\
\text { Indicadores de: } \\
\text { 2.2.1 Educación y formación profesional. } \\
\text { 2.2.2 Habilidades profesionales y formación general. }\end{array}$ & $\begin{array}{l}\text { 3.1 Requisitos para obtener la calificación de } \\
\text { contador profesional. } \\
\text { Normas referentes: } \\
\text { 3.1.1 IES 1.-Requerimientos de Ingreso a los Progra- } \\
\text { mas de Educación Profesional en Contaduría. } \\
\text { 3.1.2 IES 2.- Desarrollo Profesional Inicial - Compe- } \\
\text { tencia Técnica. } \\
\text { 3.1.3 IES 3.- Desarrollo Profesional Inicial - Habilida- } \\
\text { des Profesionales. } \\
\text { 3.1.4 IES 4.- Desarrollo Profesional Inicial - Valores, } \\
\text { Ética y Actitud Profesionales. } \\
\text { 3.1.5 IES 5.- Desarrollo Profesional Inicial - Experien- } \\
\text { cia Práctica. } \\
\text { 3.1.6 IES 6.- Desarrollo Profesional Inicial - Evalua- } \\
\text { ción de la Competencia Profesional. }\end{array}$ \\
\hline $\begin{array}{l}\text { 1.2 Formación posterior a la gradua- } \\
\text { ción: } \\
\text { 1.2.1 Educación continua. } \\
\text { 1.2.2 Formación del personal por parte de } \\
\text { empresas y firmas de auditoría. } \\
\text { 1.2.3 Obtención de cualificación profesional. }\end{array}$ & $\begin{array}{l}\text { 2.3 Plan de estudios de contabilidad global y otros } \\
\text { requisitos de calificación: } \\
\text { 2.3.1 Evaluación de competencia profesional. } \\
\text { 2.3.2 Experiencia práctica. } \\
\text { 2.3.3 Educación profesional continua. } \\
\text { 2.3.4 Esquema de certificación. } \\
\text { 2.4 Instrumento de Desarrollo Contable (Pilar C } \\
\text { referido a capacidad humana). } \\
\text { Indicadores de: } \\
\text { 2.4.1 Evaluación de las capacidades y competencias } \\
\text { contables. } \\
\text { 2.4.2 Requisitos de experiencia práctica. } \\
\text { 2.4.3 Desarrollo profesional continuo. } \\
\text { 2.4.4 Formación especializada. } \\
\text { 2.4.5 Requisitos para los técnicos contables. }\end{array}$ & $\begin{array}{l}\text { 3.2 Condiciones relativas al desarrollo profesio- } \\
\text { nal continuo. } \\
\text { Norma referente: } \\
\text { 3.2.1 IES 7.- Desarrollo Profesional Continuo } \\
\text { 3.3 Requisitos de competencia de auditores pro- } \\
\text { fesionales. } \\
\text { Norma referente: } \\
\text { 3.3.1 IES 8.- Desarrollo Profesional para Socios del } \\
\text { Trabajo, Responsables de Auditorías de Estados Fi- } \\
\text { nancieros. }\end{array}$ \\
\hline
\end{tabular}

Los pilares son: el marco legal y reglamentario, el marco institucional, la capacidad humana y el proceso de fomento de la capacidad.

De este instrumento, nos concierne el pilar $\mathrm{C}$ relativo a evaluar el desarrollo de capacidad humana, el cual contempla medir si los programas de educación profesional en contabilidad incluyen ciertos conocimientos básicos.

La Tabla 4 permite identificar un alto nivel de coincidencia en los conocimientos que la UNCTAD, ISAR y IAESB proponen o evalúan en un programa de contaduría (Tabla 4, incisos 2.1.2, 2.2.1 y 3.1 .2 , respectivamente). Sin embargo, aunque se están mencionando selectivamente los contenidos más relevantes, se puede apreciar que el plan de estudios que propone la UNCTAD es más detallado, amplio y requiere de ciertos conocimientos en un nivel avanzado. Adicionalmente, propone incorporar experiencia práctica en los estudiantes a través de internships.

Cabe señalar que el MC de la UNCTAD no incluye requerimientos para ingresar a un programa de educación en contaduría como sí lo hace la IES 1 del IAESB; sin embargo, el IDC contempla una pregunta sobre el cumplimiento de los requisitos de ingreso, tal como lo propone el IAESB.

4 Fuente: Patiño \& Valero (2013) a partir de IFAC (2010). 
Tabla 5. Contenido de programas de estudio en contaduría sugeridos por organismos internacionales ${ }^{5}$

\begin{tabular}{|c|c|c|}
\hline UNCTAD (MC) & $\begin{array}{c}\text { ISAR (IDC) } \\
\text { [Pilar C, indicadores C.1 y C.2] }\end{array}$ & $\begin{array}{l}\text { IAESB (IES) } \\
\text { [IES 2] }\end{array}$ \\
\hline $\begin{array}{l}\text { Conocimiento organizacional y de negocios: } \\
\text { - Economía. } \\
\text { - Métodos cuantitativos y estadística para nego- } \\
\text { cios. } \\
\text { - Políticas de negocios generales, estructura y } \\
\text { comportamiento organizacional. } \\
\text { - Funciones administrativas. } \\
\text { - Mercadotecnia. } \\
\text { - Negocios internacionales. }\end{array}$ & $\begin{array}{l}\text { - Economía. } \\
\text { - Entorno de los negocios. } \\
\text { - } \text { Cétodos cuantitativos. } \\
\text { - Gobernanza corporativa. } \\
\text { - Ética de la empresa. } \\
\text { - Mercados financieros. } \\
\text { - Administración y toma de decisiones estra- } \\
\text { - } \text { tégicas. } \\
\text { - Nercadeo. }\end{array}$ & $\begin{array}{l}\text { Estipula áreas de competencia en: } \\
\text { Gobierno corporativo, administración del riesgo } \\
\text { y control interno. } \\
\text { Nivel mínimo de competencia: intermedio. } \\
\text { Entorno de negocios y organizacional. } \\
\text { Nivel mínimo de competencia: intermedio. } \\
\text { Economía. } \\
\text { Nivel mínimo de competencia: fundamentos. } \\
\text { Administración de negocios. } \\
\text { Nivel mínimo de competencia: intermedio. }\end{array}$ \\
\hline $\begin{array}{l}\text { Tecnologías de información (TI): } \\
\text { - Conceptos de TI para sistemas de negocios, } \\
\text { recursos de TI para funciones contables. } \\
\text { - Control interno en sistemas de negocios basa- } \\
\text { - dos en computadora. } \\
\text { - Adopción, implementación y uso de TI. } \\
\text { - Costión de seguridad informática. } \\
\text { Comercio electrónico. }\end{array}$ & $\begin{array}{l}\text { - Conocimiento general de la TI. } \\
\text { - Conocimiento del control de la TI. } \\
\text { - Competencias del control y del usuario de } \\
\text { la TI. } \\
\text { - Una competencia o mezcla de competencias } \\
\text { de las funciones gerenciales, de evaluación } \\
\text { y de diseño de los sistemas de información. }\end{array}$ & $\begin{array}{l}\text { Tecnología de la información. } \\
\text { Nivel mínimo de competencia: intermedio. }\end{array}$ \\
\hline $\begin{array}{l}\text { Conocimientos básicos de contabilidad, audi- } \\
\text { toría, impuestos y afines a la contabilidad: } \\
\text { - Contabilidad básica: identificar, medir y comu- } \\
\text { nicar transacciones económicas y financieras } \\
\text { - usando IFRS. } \\
\text { - Contabilidad financiera: preparar estados finan- } \\
\text { cieros aplicando IFRS. } \\
\text { - Contabilidad financiera avanzada. } \\
\text { Contabilidad Administrativa } \\
\text { - Métodos y sistemas de costeo. } \\
\text { - Fijación de precios de bienes y servicios. } \\
\text { - Presupuestos. } \\
\text { - Costeo estándar. } \\
\text { Impuestos } \\
\text { - Sistema de fiscalización nacional. } \\
\text { - Tributación de empresas incorporadas. } \\
\text { - Planeación fiscal. } \\
\text { - Esquemas de seguridad social. } \\
\text { Sistemas de información contable (SIC): } \\
\text { - Desarrollo de estándares y prácticas para SIC. } \\
\text { - Almacenamiento de base de datos. }\end{array}$ & $\begin{array}{l}\text { - Contaduría financiera y presentación de in- } \\
\text { - Cormes. } \\
\text { - } \text { Contaduría gerencial y control de gestión. } \\
\text { - } \text { Derecho mercantil y societario. } \\
\text { - Auditoría y aseguramiento. } \\
\text { - Finanzas y administración financiera. } \\
\text { - Valores y ética profesionales. } \\
\text { - Auditorías de información financiera históri- } \\
\text { ca de nivel avanzado que incluye: prácticas } \\
\text { óptimas de auditoría, temas actuales de im- } \\
\text { portancia, las NIA y demás normas o leyes } \\
\text { aplicables. } \\
\text { - Contabilidad financiera y presentación de } \\
\text { informes de nivel avanzado que incluye las } \\
\text { IFRS. } \\
\text { - Sistemas de tecnología de la información } \\
\text { para la contabilidad financiera y la presenta- } \\
\text { ción de informes. } \\
\text { - Evaluación de controles y de riesgos en los } \\
\text { sistemas de contabilidad y presentación de } \\
\text { informes. }\end{array}$ & $\begin{array}{l}\text { Contabilidad financiera e información: } \\
\text { - } \quad \text { Aplicar IFRS. } \\
\text { - Elaborar estados financieros básicos y consolida- } \\
\text { dos, etc.). Nivel mínimo de competencia: avanzado. } \\
\text { Contabilidad administrativa } \\
\text { - Costeo de productos, análisis de variaciones, admi- } \\
\text { - nistración de inventarios y presupuestos. } \\
\text { - Integrar información financiera y no financiera para } \\
\text { proporcionar información relevante para la toma de } \\
\text { decisiones gerencial } \\
\text { - Preparar reportes de presupuesto, administración } \\
\text { de costos, control de calidad, medición del desem- } \\
\text { - peño, y puntos de referencia } \\
\text { - Nivel mínimo de competencia: intermedio } \\
\text { Impuestos: } \\
\text { - Requisitos de cumplimiento y presentación de im- } \\
\text { - } \text { cálculos nacionales } \\
\text { indirectos de personas y organizaciones } \\
\text { - Asuntos de impuestos relacionados con transaccio- } \\
\text { - } \text { nes internacionales no complejas } \\
\text { tos, disminución de impuestos, y evasión de im- } \\
\text { - } \text { puestos } \\
\text { Nivel mínimo de competencia: intermedio }\end{array}$ \\
\hline
\end{tabular}

5 Fuente: Elaboración propia a partir de UNCTAD (2011), UNCTAD-ISAR (2010) y IAESB (2013). 


\begin{tabular}{|c|c|}
\hline $\begin{array}{l}\text { Derecho de los negocios: } \\
\text { - Sistema legal nacional. } \\
\text { - Principios generales de leyes de contratos } \\
\text { (contratos laborales, etc.). } \\
\text { - Leyes de las empresas. } \\
\text { - Ética de negocios. } \\
\text { Fundamentos de auditoría y aseguramiento: } \\
\text { - Marco conceptual regulatorio de auditoría y es- } \\
\text { - tándares internacionales de auditoria. } \\
\text { - Evaluación y planeación de una auditoría. } \\
\text { Finanzas empresariales y administración finan- } \\
\text { ciera: } \\
\text { - Objetivos financieros por tipo de empresa y } \\
\text { marco conceptual de la administración finan- } \\
\text { - ciera. } \\
\text { - } \text { Fuentes de financiamiento (decisiones de fi- } \\
\text { - nanciamiento). } \\
\text { - Desembolsos de capital (decisiones de inver- } \\
\text { sión). } \\
\text { Integración de conocimientos }\end{array}$ & 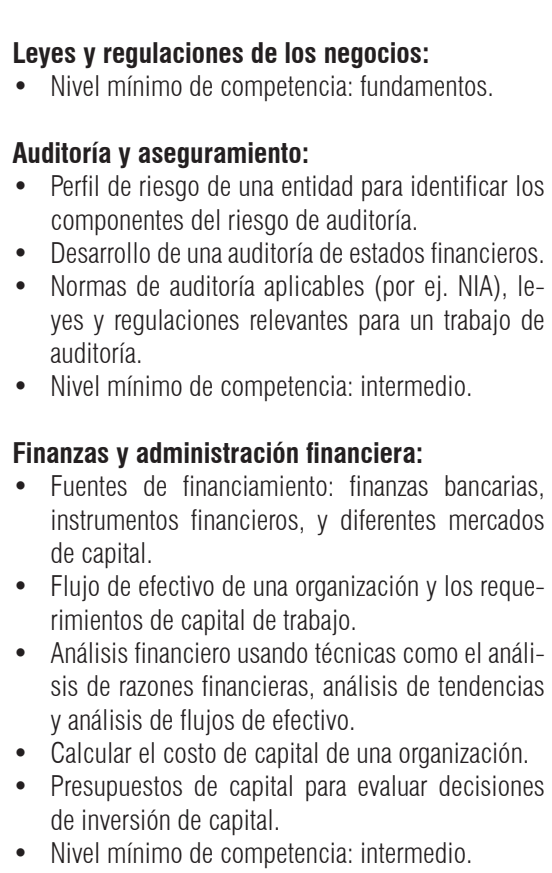 \\
\hline $\begin{array}{l}\text { Contabilidad avanzada, finanzas y tópicos re- } \\
\text { lacionados: } \\
\text { - Contabilidad financiera avanzada y reportes de } \\
\text { industrias especializadas. } \\
\text { - Tópicos avanzados de Contabilidad adminis- } \\
\text { trativa, Impuestos, Derecho de los negocios, } \\
\text { Auditoría y Finanzas. } \\
\text { - Prácticas contables (internship). }\end{array}$ & \\
\hline
\end{tabular}

En el caso de México, la ANFECA (2011) en su carácter de representante académico de las universidades, ha planteado que una Licenciatura en Contaduría Pública debe contener tres áreas fundamentales: formación general (conocimientos de administración, contabilidad básica, ética, derecho, estadística, economía, etc.); formación sustantiva profesional (conocimientos de contabilidad, fiscal, costos, auditoría, finanzas, jurídicos, informática, etc.); y formación integral profesional que contempla temas de auditoría interna, operacional y gubernamental, contabilidad internacional, evaluación de proyectos de inversión, y la posibilidad de que los estudiantes cursen cuatro asignaturas optativas.

Adicionalmente, el Instituto Mexicano de Contadores Públicos, a través de su Comisión de Educación, emitió en el año 2009 un proyecto de Normas de
Formación para Profesionales en Contaduría con la finalidad de proveer una guía a las instituciones educativas de forma tal que se homologaran sus planes de estudio (CCPM, 2009). En la actualidad, dicha comisión ha decidido adoptar las IES que emite el IAESB y tiene contemplado en su plan de trabajo, elaborar guías para llevar a cabo su implementación.

En Colombia el (Decreto 939, 2002), reglamenta que los programas de Contaduría Pública deben incluir: un área de formación básica, la cual incluye matemáticas, estadística, economía, entre otros y un área de formación profesional con cuatro componentes: ciencias contables y financieras, formación organizacional, información y regulación. Adicionalmente, se enuncia la obligatoriedad de tener componentes flexibles en los planes de estudios y que los programas deben estar estructurados por créditos académicos. 
En un estudio previo (Patiño \& Santos, 2009) sobre planes de estudios de programas de Contaduría Pública en Colombia se llegó a la conclusión de que los contenidos curriculares son muy diversos, sin embargo, las áreas más representativas en términos porcentuales son: contabilidad financiera, economía, área cuantitativa, finanzas y control en ese orden.

Continuando con el comparativo presentado en la Tabla 4, se identifican puntos de coincidencia entre los incisos 2.2 .2 y 3.1.3, planeados por UNCTADISAR y IAESB respectivamente, relativos con las habilidades profesionales.

Por su parte, el cuestionario (IDC) que está aplicando la UNCTAD-ISAR en ciertos países, contiene indicadores que evalúan las habilidades intelectuales que promueve un programa de contaduría. Estos indicadores se encuentran en el pilar $\mathrm{C}$ de dicho cuestionario, indicador C.3. Tales habilidades están relacionadas con la capacidad de comprender, aplicar, analizar, sintetizar y evaluar temas contables y de auditoría, combinando conocimientos de varias áreas. Adicionalmente están incluidas habilidades técnicas y funcionales relacionadas con las matemáticas, estadística, dominio de tecnologías de la información y modelos de decisión y análisis de riesgo; así como habilidades personales de autogestión, iniciativa, escepticismo profesional, trabajo en equipo, negociación, liderazgo, entre otras (UNCTAD-ISAR, 2010).

El IAESB por su parte, a través de la propuesta de norma IES 3: Desarrollo Profesional Inicial - Habilidades Profesionales, decidió adoptar el enfoque de resultados del aprendizaje para describir la competencia profesional de esta norma, en lugar de establecer una lista de áreas de habilidades. Dicha norma categoriza las habilidades profesionales en cuatro áreas de competencia (IAESB, 2013):

- Habilidades intelectuales que se relacionan con la capacidad de un contador profesional para resolver problemas, tomar decisiones, y ejercer buen juicio (nivel mínimo de competencia: avanzado).

- Habilidades personales que se relacionan con las actitudes y conducta personales de un conta- dor profesional (nivel mínimo de competencia: intermedio).

- Habilidades interpersonales y de comunicación que se relacionan con la capacidad de un contador profesional para trabajar en forma efectiva con otros (nivel mínimo de competencia: intermedio).

- Habilidades organizacionales que se relacionan con la capacidad de un contador profesional para trabajar de manera efectiva con o dentro de una organización para obtener lo mejor de la gente y recursos disponibles (nivel mínimo de competencia: intermedio).

Las propuestas del UNCTAD-ISAR y del IAESB en cuanto a las habilidades profesionales tienen una alta convergencia, aunque con un enfoque distinto. En el primer caso se establece una lista de habilidades, mientras que en el segundo se formulan competencias.

En cuanto a los temas de valores, ética y actitud profesionales, el mismo indicador C.3 del IDC incluye parámetros para evaluar si el componente de ética está incluido en la formación profesional. El cuestionario evalúa entre otros aspectos, el cumplimiento con los principios éticos fundamentales de integridad, objetividad, compromiso con la competencia profesional y la confidencialidad. También el comportamiento profesional, así como la ética relacionada con la profesión (responsabilidad social) y las leyes (interés público) (UNCTAD-ISAR, 2010).

La IES 4 (inciso 3.1.4 de la Tabla 4) requiere que un programa en contaduría incluya cinco principios fundamentales de ética profesional: Integridad; Objetividad; Competencia Profesional y Debido Cuidado; Confidencialidad; y Conducta Profesional. Debido al enfoque por competencias, la norma establece los resultados del aprendizaje que demuestren los valores, ética y actitud profesionales, de acuerdo con las siguientes áreas de competencia (IAESB, 2013):

- Escepticismo profesional y juicio profesional (nivel mínimo de competencia: intermedio). 
- Principios éticos (nivel mínimo de competencia: intermedio).

- Compromiso con el interés público (nivel mínimo de competencia: intermedio).

La IES 5 (inciso 3.1 .5 de la Tabla 4), especifica los requerimientos de la experiencia práctica durante el desarrollo profesional inicial (formación académica hasta la obtención de la graduación universitaria). La norma señala que sean los organismos miembros del IFAC, en el caso de México, el Instituto Mexicano de Contadores Públicos (IMCP), los que requieran la experiencia práctica suficiente a los aspirantes a contadores profesionales. Dicha experiencia puede ser bajo tres enfoques: basado en resultados, en conocimiento o en una combinación de ambas (IAESB, 2013).

En la realidad mexicana, este requerimiento no lo señala el IMCP, sino que es una elección de las instituciones educativas, solicitar tal experiencia.

En el caso de Colombia no se encuentra explicito el tema, por lo menos en los contenidos mínimos dados por la ley, sin embargo, la (Ley 43 de 1990), se incluyen los aspectos referentes a la ética y en este sentido existe la discusión de si se debe incluir en el plan de estudios como asignatura o como contenido transversal, sin embargo no se enuncia nada relacionado con la experiencia al respecto.

Finalmente, se termina de describir la Tabla 4, señalando que la IES 6 (inciso 3.1.6 de la Tabla 4), establece que los organismos miembros del IFAC evalúen áreas específicas de competencia profesional de los aspirantes a contadores, durante su desarrollo profesional a través de evidencias que pueden ser exámenes escritos u orales, pruebas objetivo, pruebas por computadora, evaluaciones de competencia en el lugar de trabajo realizadas por empleadores o revisión de un portafolio de evidencias sobre la finalización de actividades en el lugar de trabajo (IAESB, 2013).

En este sentido, la UNCTAD-ISAR tanto en su propuesta de plan de estudio de contabilidad global de 1999, como en el cuestionario IDC (Tabla 4, incisos 2.1.3 y 2.2.3), consideran la evaluación final de competencias profesionales, sugiriendo que también sea el organismo profesional el que se encargue de su realización al final de su formación educativa (UNCTAD-ISAR, 1999; 2010).

En México el IMCP no tiene facultades para realizar alguna evaluación como lo señala la IES 6; sin embargo existe un organismo independiente llamado Centro Nacional de Evaluación para la Educación

Tabla 6. Análisis comparativo de las características del subsistema de formación contable con los lineamientos internacionales posterior a la graduación ${ }^{6}$

\begin{tabular}{|c|c|c|}
\hline Jarne (1997) & UNCTAD & IAESB \\
\hline $\begin{array}{l}\text { 1.2 Formación posterior a la graduación: } \\
\text { 1.2.1 Educación continua. } \\
\text { 1.2.2 Formación del personal por parte de } \\
\text { empresas y firmas de auditoría. } \\
\text { 1.2.3 Obtención de cualificación profesional. }\end{array}$ & $\begin{array}{l}\text { 2.1 Plan de estudios de contabilidad global y otros } \\
\text { requisitos de calificación: } \\
\text { 2.1.4 Experiencia práctica. } \\
\text { 2.1.5 Educación profesional continua. } \\
\text { 2.1.6 Esquema de certificación. } \\
\text { 2.2 Instrumento de Desarrollo Contable (Pilar C re- } \\
\text { ferido a capacidad humana). } \\
\text { Indicadores de: } \\
\text { 2.2.4 Requisitos de experiencia práctica. } \\
\text { 2.2.5 Desarrollo profesional continuo. } \\
\text { 2.2.6 Formación especializada. } \\
\text { 2.2.7 Requisitos para los técnicos contables. }\end{array}$ & $\begin{array}{l}\text { 3.2 Condiciones relativas al desarrollo profe- } \\
\text { sional continuo. } \\
\text { Norma referente: } \\
\text { 3.2.1 IES 7.- Desarrollo Profesional Continuo. } \\
\text { 3.3 Requisitos de competencia de auditores } \\
\text { profesionales. } \\
\text { Norma referente: } \\
\text { 3.3.1 IES 8.- Desarrollo Profesional para Socios del } \\
\text { Trabajo, Responsables de Auditorías de Estados Fi- } \\
\text { nancieros. }\end{array}$ \\
\hline
\end{tabular}

6 Fuente: Elaboración propia de acuerdo con Jarne (1997), UNCTAD-ISAR (1999; 2010) y UNCTAD (2013). 
Superior, A.C. cuya actividad principal es el diseño y la aplicación de instrumentos de evaluación de conocimientos, habilidades y competencias, así como el análisis y la difusión de los resultados que arrojan las pruebas que elabora (CENEVAL, 2013). El Examen General de Egreso de la Licenciatura (EGEL) en Contaduría tiene por objetivo identificar si los egresados de dicha licenciatura cuentan con los conocimientos y habilidades necesarios para iniciarse eficazmente en el ejercicio de la profesión.

En Colombia no existen evaluaciones profesionales obligatorias, esta es la principal diferencia con el caso mexicano, lo cual ha sido cuestionado constantemente ya que no existe ninguna garantía de que los profesionales contables tengan las cualidades necesarias para desempeñar su profesión.

Una vez terminada la formación académica de un contador público, las tres fuentes de referencia, señalan como características principales de esta etapa, la actualización constante en la profesión a través de la educación continua y la certificación o cualificación profesional.

En cuanto a la educación continua, la IES 7 (Tabla 6, inciso 3.2.1) promueve que los organismos miembros del IFAC requieran el desarrollo profesional continuo como un componente integral requerido a un contador público para que sea miembro del organismo profesional.

Por su parte, la UNCTAD-ISAR (1999; 2010) también proponen y evalúan este aspecto (Tabla 6 , incisos 2.1.5 y 2.2.5). El IDC cuestiona si es el organismo profesional, las universidades o un órgano gubernamental quien ofrece cursos de desarrollo profesional continuo (DPC). También cuestiona si hay un número mínimo de horas o unidades de aprendizaje que cumplir, si se usa un enfoque basado en resultados para evaluar el DPC, si hay un procedimiento para evaluar el tipo de cursos y si la certificación como contador se puede cancelar en caso de no cumplir con un número adecuado de horas de DPC.

En el caso de México, el Instituto Mexicano de Contadores Públicos es el encargado de evaluar el cumplimiento del DPC para lo cual tiene establecida una
Norma de Educación Profesional Continua (NEPC) requiriendo que los miembros del instituto, presenten evidencias de aprendizaje permanente. De acuerdo con el sector profesional en el que se desempeñe el contador público, debe cumplir con un mínimo de horas (puntos) cada año calendario, para dar cumplimiento a la NEPC. El IMCP revisa que los cursos de capacitación correspondan al área de la profesión y cuenta con un registro nacional de capacitadoras para validar a las instituciones que otorgan cursos (IMCP, 2013).

En el caso de Colombia no se garantiza que los contadores se estén formando constantemente, un tema tan importante como la educación continua es voluntario y aunque se ha dinamizado constantemente por el proceso de convergencia hacia IFRS, este no puede ser un proceso temporal, es contemplado este tema solamente en el código de ética de la profesión.

La IES 8 establece los requerimientos de desarrollo profesional para aspirantes a socios del trabajo y para socios del trabajo en funciones, que presten servicios de aseguramiento para auditorías de estados financieros (IAESB, 2013).

Para Jarne (1997) y la UNCTAD-ISAR (1999; 2010), dicha norma está relacionada con la certificación del contador público, pues en varios países, como es el caso de México, la certificación es requisito para auditar y dictaminar estados financieros. La UNCTADISAR en el IDC, cuestiona si en el país se exige periodo fijo de experiencia mínimo de tres años para poder obtener la calificación (certificación), así como si existe un organismo que supervise dicha experiencia (UNCTAD-ISAR, 2010).

En la realidad mexicana, para poder aspirar a la certificación o cualificación profesional, es requisito contar con el título profesional, tener al menos tres años de experiencia en actividades relacionados con la Contaduría Pública y presentar un Examen Uniforme de Certificación que elabora el propio IMCP, en diversos campos de especialización de la profesión (IMCP, 2013).

En Colombia la certificación profesional se obtiene con un año de experiencia profesional certificada 
por una organización legalmente constituida, la cual puede cumplirse ejerciendo labores de investigación (Junta Central de Contadores, s.f.).

\section{Conclusiones}

En primera estancia se debe mencionar la importancia que tiene la formación contable en el desarrollo de la disciplina, de la profesión y por ende en el apoyo al desarrollo social del entorno. El subsistema de formación es el encargado de formar profesionales con las calidades para responder a los procesos vigentes y ante las necesidades tan palpables de las economías latinoamericanas.

Un concepto relevante que aparece con los modelos de regulación en formación contable de nivel internacional es el de competencia, el cual ha sido desarrollado en forma general en todas las disciplinas $y$ todos los países, lo que se ha prestado para críticas en cuanto a la homogenización en la educación y por lo tanto en los enfoques de los profesionales, básicamente tendiendo hacia lo técnico.

En los dos subsistemas de formación que se están comparando (Colombia y México), existen diferencias muy relevantes, ya que las realidades respecto a las situaciones de los países han sido muy distintas, dentro de estos contrastes se resaltan: El modelo mexicano se asimila más en la estructuración de los planes de estudio a lo contenido en las propuestas de organismos internacionales, sin embargo se coincide en lo contemplado en la norma colombiana en lo referente a la incorporación de un área básica y una profesional. Otra diferencia puntual aparece con la presentación obligatoria de examen de certificación en México y no en Colombia, ya que este aspecto impacta directamente en las calidades de los profesionales contables.

Por otra parte se coincide en que no es obligatorio el componente de prácticas profesionales dentro de los planes de estudio, aspecto que es negativo ya que dicho proceso le da al estudiante otros puntos de vista y experiencias distintas a la formación dentro del aula, otro aspecto relevante es la ausencia de contenidos en temas investigativos, lo cual le da una visión distinta al profesional, siendo más analítico y crítico.
En resumen, la descripción de los subsistemas de formación en los países de estudio, sugiere que un mayor involucramiento de la iniciativa privada en México, como es el caso del CENEVAL y el IMCP (miembro del IFAC), ha generado la implementación de prácticas que son recomendadas por los organismos internacionales; tal es el caso de un examen de egreso a los estudiantes de la licenciatura en contaduría, un examen de certificación para los profesionales y una exigencia de educación profesional continua. Esto aunado a la influencia también privada, de organismos como el CINIF, entidad emisora independiente al gobierno, encargada de elaborar normas contables locales. Situación contraria a lo que ha sucedido en Colombia donde se sigue discutiendo la conveniencia de exigir exámenes de certificación profesional y no se exige actualización profesional.

Por último, es importante resaltar la necesidad de incluir propuestas educativas que no solo contemplen temáticas necesarias en los planes de estudio sino consistencia en los modelos formativos hacia la elaboración de currículos con una misión y visión clara que incluyan contenidos interdisciplinarios, de investigación, entre otros.

\section{Referencias}

Asociación Interamericana de contabilidad - AIC (2013). . Recuperado el 10 de diciembre de 2013 de: http://www.contadores-aic.org/index.php?node $=920$

Asociación Nacional de Facultades y Escuelas de Contaduría y Administración - ANFECA (2011). Ponencia central: "Hacia un espacio común de educación superior en las facultades y escuelas de negocios en México". Recuperado el 16 de mayo de 2013 de: http://www.anfeca.unam.mx/inicio.php

Callao, S. \& Jarne, J. (1995). La información financiera en el contexto internacional: Análisis descriptivo. En: Revista Española de Financiación y Contabilidad: 937-969.

Cañibano, L. \& Mora, A. (1999). Variables explicativas de los sistemas de regulación contable: influencia de la profesión en los países de la Unión Europea. En: Revista Española de Financiación y Contabilidad: 291-326.

Chiquiar, W. (2010). El modelo contable internacional y el proceso de convergencia a las normas internacionales - el desafío de la República Argentina. Conferencia presentada en la Universidad Nacional de Salta en Agosto 2010. Recuperado el 05 de diciembre de 2013 de: http://unq.academia.edu/wrc/Papers

Centro Nacional de Evaluación para la Educación Superior - CENEVAL (2013). . Recuperado el 10 de diciembre de 2013 de: http://www.ceneval.edu.mx/

Colegio de Contadores Públicos de México - CCPM (2009). Normas de formación para profesionales en contaduría. Anexo 1. 
Recuperado el 23 de mayo de 2013 de: http://www.ccpm.org. $\mathrm{mx} /$ avisos/AnexoFolio12.pdf

Decreto 939 de 2002 "Por el cual se establecen estándares de calidad en programas profesionales de pregrado en Contaduría Pública". (16 de mayo de 2002). Bogotá, Colombia.

Doupnik, T. \& Salter, S. (1993). An Empirical test of a judgmental international classification of financial reporting. En: Journal of International Business Studies: 41-60.

García, M. \& Gandía, J. (1998). Análisis del grado de armonización Europeo basado en los sitemas contables. En: Revista Española de Financiación y Contabilidad: 951-978.

García, M., Laínez, J. \& Monterrey, J. (1995). La investigación en Contabilidad Internacional: Una visión panóramica. En: Revista Española de Financiación y Contabilidad: 337-363.

García, M. \& Garrido, P. (1995). Los sistemas contables y la armonización Europea. En: Revista Española de Financiación y Contabilidad: 991-1013.

IAESB (2013). Estándares Internacionales de Formación en Contaduría. Recuperado el 07 de diciembre de 2013 de: https:/www.ifac. org/publications-resources/international-education-standard-ies

Instituto Mexicano de Contadores Públicos - IMCP (2013). . Recuperado el 10 de diciembre de 2013 de: http://imcp.org.mx/norma-epc

Jarne, J. (1997). Clasificación y evolución internacional de los sistemas contables. Madrid: Asociación Española de Contabilidad y Administración de Empresas - AECA.

Joshi, S., Takara, R., Gremone, L. \& González, B. (2012). América Latina está en el camino de las IFRS ¿Seguirá con la misma velocidad o la reducirá? Standard \& Poor's.

Junta Central de Contadores (s.f.). Requisitos tarjeta profesional. Disponible en: http://jccconta.gov.co/index.php?option=com_co ntent\&view $=$ article\&id $=95 \&$ Itemid $=280$

Laínez, J. \& Jarne, J. (1999). El entorno como variante discriminante en la diferenciación internacional de sistemas contables. En: Revista de Contabilidad: 73-101.

Laínez, J., Callao, G., Gandía, J., García, M., Jarne, J. \& Monterrey, J. (2001). Manual de contabilidad internacional. Madrid: Pirámide.

Martín, J. (2000). La formación universitaria en contabilidad. En: Partida doble, 113: 72-83.

Martínez, G. (2007). La educación contable en el nuevo contexto de significación. En: Contaduría Universidad de Antioquia, 50: 43-76.

Montoya, J. \& Farías, G. (2011). Desarrollo de habilidades profesionales y adquisición de conocimientos en los programas académicos de Administración de Empresas y Contaduría
Pública: una investigación exploratoria con estudiantes de España y México. En: INNOVAR, 21(40): 93-106.

Morales, F. \& Jarne, J. (2006). Clasificación internacional de los sistemas contables de Argentina, Brasil y Chile. En: Panorama Socio-económico: 90-95.

Patiño, R. \& Santos, G. (2009). Planes de estudio de Contaduría Pública en Colombia y las propuestas de formación profesional. En: Revista Internacional Legis de Contabilidad \& Auditoría, 37: 131-163.

Patiño, R. \& Valero, G. (2013). Competencias de los estudiantes de Contaduría Pública: aproximación desde lo institucional. IX CEAC. Punta de Este, Uruguay.

Patiño, R. \& Vásquez, N. (2013). Las características de los subsistemas contables de Colombia y México ante la convergencia a Normas Internacionales de Información Financiera. En: Cuadernos de Contabilidad, 37.

Ramírez, D. (2013). Contabilidad Administrativa. (9a . Ed.) México: Mc GrawHill.

Rodríguez, P. (s.f.). Contabilidad internacional. Recuperado el 04 de 04 de 2013, de http://www.panorama.utalca.cl/dentro/sdd/ conta inter.pdf

UCTAD-ISAR (1999). Guideline for a global accounting curriculum and other qualification requirements. TD/B/COM.2/ISAR/5. Recuperado el 07 de diciembre de 2013 de: http://unctad.org/ en/Docs/c2isard5.en.pdf

UNCTAD-ISAR (2010). Capacity-building framework for highquality corporate reporting: assessment questionnaire. TD/B/C. II/ISAR/66. Recuperado el 07 de diciembre de 2013 de: http:// unctad.org/en/Docs/ciiisard56 en.pdf

UNCTAD (2011). Model Accounting Curriculum (Revised). UNCTAD/ ITE/EDS/9. United Nations.

UNCTAD (2013). Bases fundamentales de la presentación de información empresarial de alta calidad: retos para el desarrollo de los recursos humanos. Recuperado el 04 de diciembre de 2013 de: http://unctad.org/meetings/es/SessionalDocuments/ ciiisard66_es.pdf

Valero, G., Patiño, R. \& Duque, O. (2013). Competencias para el programa de Contaduría Pública: una aproximación conceptual. En: Revista Contaduría. Universidad de Antioquia, (62): 11-36.

Vásquez, N. (2010). Análisis del proceso de adopción de IFRS en México. En: Revista Contaduría. Universidad de Antioquia, 57: 51-67

Zorio, A., García, M. \& Pucheta, M. (2004). Calidad, un nuevo concepto clave. En: Revista Española de Financiación y Contabilidad: 313-347. 